\section{P76 SURVEILLANCE GUIDELINES: LONG-TERM ADENOMA INCIDENCE FOLLOWING POLYP EXCISION AT INDEX COLONOSCOPY}

\author{
1,2,3 Anisha Sukha*, ${ }^{2}$ Jacob Househam, ${ }^{2}$ Kit Curtius, ${ }^{2}$ Trevor Graham, ${ }^{1,3}$ Adam Humphries. \\ ${ }^{1}$ Wolfson Unit for Endoscopy, St Mark's Hospital, London, UK; ${ }^{2}$ Barts Cancer Institute, \\ London, UK; ${ }^{3}$ Imperial College London, London, UK
}

\subsection{6/gutjnl-2020-bsgcampus. 151}

Introduction 1 in 10 people in the UK have a detectable colorectal adenoma. Most adenomas are asymptomatic and detected incidentally during national screening and surveillance programmes. Post-polypectomy, these patients are considered to be at an increased risk of colorectal cancer (CRC), with calculations primarily based on polyp size and multiplicity.

To investigate the outcomes of endoscopic surveillance and compare the British Society of Gastroenterology (BSG) 2012 guidelines with the recently revised 2019 guidelines. The primary outcome was to determine the incidence of advanced adenomas on repeat investigations following the complete excision of all polyps at the index procedure.

Methods Post-polypectomy patients under surveillance at a single large tertiary referral centre serving a secondary care population of approximately 1 million were identified. Patients with a familial hereditary condition, polyposis syndrome, inflammatory bowel disease (IBD), previous CRC diagnosis and history of bowel resection were excluded. Patients were categorised as 'high,' 'intermediate' or 'low' risk using the 2012 BSG guidelines and re-categorised as 'large non-pedunculated colorectal polyp (LNPCP),' 'high risk,' or 'discharge' using the 2019 BSG guidelines. Patients were assigned an advanced Polyp Index (API) score following each endoscopy $($ size $\geq 10 \mathrm{~mm}=1$, number $\geq 5=1$, high-grade dysplasia $=2$ ) for analyses.

Results Between October 2011 and Dec 2014, 1190 patients $(\mathrm{M}=816(69 \%)$, mean age $=62)$ had a primary colonoscopy and polypectomy. During surveillance, $n=2(0.19 \%)$ CRCs were detected (mean interval $=3.2$ years). Patients were stratified into high $(n=484)$, intermediate $(n=303)$ and low $(n=403)$ risk groups and follow-up data was collected until December 2019 , mean $=7.6[\mathrm{SD}= \pm 0.9]$ years.

API $\geq 1$ during surveillance was detected in $n=46(9.5 \%)$, $\mathrm{n}=11(3.6 \%), \mathrm{n}=7(1.7 \%)$ patients in high, intermediate and low risk patients respectively, as per 2012 guidelines; high risk vs intermediate risk $(\mathrm{p}=0.0017)$, intermediate risk vs low risk $(\mathrm{p}=0.146)$. On re-stratification to 2019 guidelines, API $\geq 1$ was determined in LNPCP $(n=177)$, high risk $(n=296)$ and discharge $(n=717)$, as $n=15 \quad(8.5 \%), n=34 \quad(11 \%)$ and $n=18$ $(2.5 \%)$ respectively.

Conclusions Using 2019 revised guidelines, 717 (60.1\%) patients would have been discharged following the index procedure and no CRC would have missed. 18 (2.5\%) patients with an $\mathrm{API} \geq 1$ would have been missed, however this was not significant (Fisher's Exact Test; $\mathrm{p}=0.42$ ). The revised BSG guideline for polyp surveillance reduces the burden of colonoscopy to patients and the healthcare system, without compromising the identification of significant pathology or CRC.

\section{P77 SIMULATION TRAINING FOR ENDOSCOPY ASSISTANTS}

Amelia Thorpe*, Paul Bailey. Nottingham University Hospitals NHS Trust, Nottingham, UK
Introduction The Joint Advisory Group (JAG) ISREE initiative in 2019 called for an improvement of safety in endoscopy, one of the recommendations was simulation training for staff. Endoscopy simulation courses have shown positive results for improving staff confidence in handling emergencies (Menabawey, $\mathrm{T}$ et al, 2018). A hospital conducted a simulation day in conjunction with the local simulation centre and the impact on was staff analysed.

Methods Training was provided to a range of staff from 3 endoscopy units. Simulations were delivered using a combination of clinical mannequins and simulated patients. Simulations consisted of: vasovagal, respiratory arrest, and cardiac arrest. Staff confidence in managing emergencies was assessed before and after the training using a 1-5 Likert scale for the following questions:

How confident do you feel...

1. that your clinical knowledge is appropriate to your level?

2. to practice within the limitations of your level?

3. that you possess sufficient strategies to raise concerns when necessary

4. that you are receptive to feedback from others?

5. that the course can teach you something new?

6. that you possess the skills and knowledge to assess and treat acutely unwell patients?

7. that you possess the skills required to communicate information to the MDT?

Questions with free text responses were also asked. Responses were divided into registered and unregistered.

Results 35 staff attended: 19 RNs, 2 ODPs, 2 Nurse Endoscopists, 1 Trainee Nursing Associate, and 11 HCAs. 100\% of registered staff and $91 \%$ of unregistered staff said they would recommend the course to a colleague. All registered staff showed an improvement in confidence, with the most improvement being communication during emergencies (Q3 +0.37 ) and clinical knowledge (Q1 +0.65). Question 6, relating to treating the acutely unwell patient, showed a -0.37 drop in scores for unregistered. Conversations in the debriefs indicated that most were not aware of potential endoscopic emergencies. Taking in to account their positive free text answers, it could be concluded that the decrease was due to staff realising their need for further training.

Change in staff responses:

Conclusions Simulation training was well received by staff and improved knowledge of emergency protocols and confidence in dealing with emergencies.

\section{REFERENCES}

1. Joint Advisory Group for GI Endoscopy, 2019. Improving Safety and Reducing Error in Endoscopy (ISREE) Implementation strategy [online]. London: Joint Advisory Group for GI Endoscopy. Available at: https://www.thejag.org.uk/Downloads/ JAG/General/Improving\%20Safety\%20and\%20Reducing\%20Error\%20in\% 20Endoscopy\%20(ISREE)\%20Implementation\%20strategy\%20v1.0.pdf [accessed Jan 2020]

2. Menabawey T, et al, 2018. Five years of HIFIVE (human factors in virtual endos copy): an endoscopic non-technical skills simulation programme. Gut, 67, 272.

\section{\begin{tabular}{|l|l}
\hline P78 SUBJECTS WHO UNDERWENT BOWEL SCOPE \\
\hline
\end{tabular} SCREENING ARE SIGNIFICANTLY MORE LIKELY TO RETURN FOBT/FIT SCREENING KITS}

Tania Baldwin, Ajay Verma*. Kettering General Hospital NHS FT, Kettering, UK 